\title{
Intrinsic Cholinergic Neurons in the Hippocampus: Fact or Artifact?
}

\author{
Jan Krzysztof Blusztajn ${ }^{1 *}$ and Jasmine Rinnofner ${ }^{2}$ \\ ${ }^{1}$ Department of Pathology and Laboratory Medicine, Boston University School of Medicine, Boston, MA, USA, ${ }^{2}$ Department \\ of Applied Life Sciences, University of Applied Sciences, Vienna, Austria
}

It is generally agreed that hippocampal acetylcholine (ACh) is synthesized and released exclusively from the terminals of the long-axon afferents whose cell bodies reside in the medial septum and diagonal band. The search for intrinsic cholinergic neurons in the hippocampus has a long history; however evidence for the existence of these neurons has been inconsistent, with most investigators failing to detect them using in situ hybridization or immunohistochemical staining of the cholinergic markers, choline acetyltransferase (ChAT) or vesicular acetylcholine transporter (VAChT). Advances in the use of bacterial artificial chromosome (BAC) transgenic mice expressing a reporter protein under the control of the genomic elements of the Chat gene (Chat-BAC mice) have facilitated studies of cholinergic neurons. Such mice show robust and faithful expression of the reporter proteins in all known cholinergic cell populations. The availability of the Chat-BAC mice re-ignited interest in hippocampal cholinergic interneurons, because a small number of such reporter-expressing cells is frequently observed in the hippocampus of these mice. However, to date, attempts to confirm that these neurons co-express the endogenous cholinergic marker ChAT, or release

OPEN ACCESS

Edited by:

Robert B. Renden,

University of Nevada Medical School,

USA

Reviewed by: Lorna Role,

SUNY Stony Brook, USA Lynn E. Dobrunz,

University of Alabama at Birmingham,

USA

${ }^{*}$ Correspondence: Jan Krzysztof Blusztajn jbluszta@bu.edu

Received: 08 February 2016 Accepted: 26 February 2016 Published: 10 March 2016

Citation: Blusztajn JK and Rinnofner J (2016) Intrinsic Cholinergic Neurons in the Hippocampus: Fact or Artifact?

Front. Synaptic Neurosci. 8:6. doi: 10.3389/fnsyn.2016.00006
ACh, have been unsuccessful. Without such confirmatory evidence it is best to conclude that there are no cholinergic neurons in the hippocampus. Similar considerations apply to other BAC transgenic lines, whose utility as a discovery tool for cell populations heretofore not known to express the genes of interest encoded by the BACs, must be validated by methods that detect expression of the endogenous genes.

Keywords: acetylcholine, choline acetyltransferase ChAT, vesicular acetylcholine transporter, hippocampus, transgenic mouse models, basal forebrain cholinergic neurons, septum, slc18a3

The hippocampus continues to fascinate neuroscientists as a brain region essential for encoding and retrieval of memory [e.g., (Bird and Burgess, 2008; Augustinack et al., 2014)]. One of the key components of the neuronal circuitry necessary for these processes is the innervation of the hippocampus by basal forebrain cholinergic neurons (BFCN), which provides modulatory input mediated by the neurotransmitter, acetylcholine (ACh; Teles-Grilo Ruivo and Mellor, 2013). A decline in BFCN function and diminished cholinergic marker expression is apparent in aged humans and animals (Sarter and Bruno, 2004; Gauthier et al., 2006; Haense et al., 2012), in patients with Alzheimer's disease (AD; Whitehouse et al., 1982; Bowen et al., 1983; Mufson et al., 2008; Grothe et al., 2012) and in animal models of AD (Savonenko et al., 2005; Payette et al., 2007; Perez et al., 2007; Goto et al., 2008; Mufson et al., 2008; Machová et al., 2010; Nikolajsen et al., 2011; Burke et al., 2013; Mellott et al., 2014). Thus, it has been postulated that abnormal cholinergic neurotransmission, due to dysfunction and/or degeneration of BFCN, 
contributes to the memory deficits seen in advanced age and in AD (Mufson et al., 2008; Grothe et al., 2012; Haense et al., 2012). Moreover, unlike the cerebral cortex, the hippocampus is characterized by a life-long capacity for neurogenesis that is essential for its normal function (Lee et al., 2012; Aimone et al., 2014), and age-related slowing of neurogenesis contributes to cognitive decline (Lee et al., 2012; Samson and Barnes, 2013; Aimone et al., 2014; Gray and Barnes, 2015). Studies in rats and mice indicate that cholinergic projections to the dentate gyrus promote adult hippocampal neurogenesis (Cooper-Kuhn et al., 2004; Mohapel et al., 2005; Van der Borght et al., 2005; Fontana et al., 2006; Kaneko et al., 2006; Kotani et al., 2006, 2008; Aztiria et al., 2007; Zhao et al., 2008; Fréchette et al., 2009; Ho et al., 2009; Narimatsu et al., 2009; Van Kampen and Eckman, 2010; Itou et al., 2011; Rennie et al., 2011). Therefore, investigations of cholinergic mechanisms in the hippocampus are motivated by both basic science- and translational objectives.

It is generally agreed that hippocampal $\mathrm{ACh}$ is produced and released exclusively by the terminals of BFCN. However, several studies performed in rodents have suggested the existence of a minor pool of intrinsic cholinergic neurons in the hippocampus. The support for this notion is based entirely on the immunohistochemical localization, within a small number of hippocampal cell bodies, of the enzyme choline acetyltransferase (ChAT) that catalyzes the synthesis of $\mathrm{ACh}$ and serves as the sine qua non marker of cholinergic neurons. As yet, no consensus on the existence of intrinsic hippocampal cholinergic neurons based on this method has been reached, because of studies that failed to show ChAT-positive neurons in the hippocampus (Armstrong et al., 1983; Ichikawa and Hirata, 1986), and because data on the distribution and morphology of the ChAT-positive hippocampal cells have been inconsistent (Clarke, 1985; Wainer et al., 1985; Frotscher et al., 1986; Matthews et al., 1987; Blaker et al., 1988; Kanaya-Ida and Ben Ari, 1989). Moreover, in situ hybridization failed to detect Chat mRNA-positive neurons in the hippocampus (Oh et al., 1992; Lauterborn et al., 1993). Once synthesized by ChAT, $\mathrm{ACh}$ is transported to the synaptic vesicles in a process catalyzed by the vesicular acetylcholine transporter (VAChT), encoded by the gene Slc18a3. The Chat and the Slc18a3 genes co-localize within the genome, forming the cholinergic gene locus (Eiden, 1998; for certain Chat transcripts, Slc18a3 may even be considered as one of their introns) and are co-expressed (Berse and Blusztajn, 1995). Therefore, VAChT constitutes another specific marker of cholinergic neurons. However, no studies that examined the immunohistochemical (Gilmor et al., 1996, 1998; Roghani et al., 1998; Schäfer et al., 1998) or in situ hybridization (Roghani et al., 1996; Ichikawa et al., 1997) localization of VAChT found cellular staining in the hippocampus. Likewise, direct comparison of in situ hybridization and immunohistochemical staining for ChAT and VAChT failed to detect positive cellular staining in the hippocampus (Ichikawa et al., 1997). Taken together, the available data based on immunohistochemical and in situ hybridization methods indicate that the presence of intrinsic cholinergic neurons in the hippocampus of mice and rats is unlikely.
In recent years, the availability of transgenic mice engineered for easy visualization of cholinergic neurons by the expression of indicator (e.g., fluorescent) proteins under the control of genomic elements surrounding the cholinergic gene locus, has generated a renewed interest in the intrinsic hippocampal cholinergic neurons because, in many cases hippocampal fluorescent protein-expressing cells have been observed. The general strategy for the construction of this type of transgenic mice, pioneered, perfected, and popularized by the GENSAT project (Gong et al., 2003, 2007; Heintz, 2004), relies on the use of bacterial artificial chromosomes (BACs) large enough to contain the entire cholinergic gene locus and surrounding genomic sequences thought to permit the locus expression exclusively in cholinergic cells. The BACs are first modified to encode a fluorescent protein (e.g., EGFP) or Cre recombinase, and are then used to generate transgenic mouse lines. Note, that depending on the $\mathrm{BAC}$ used, these types of transgenic mice may express additional genes that are present in the BAC together with the gene of interest (so-called "passenger" genes; Ting and Feng, 2014). Many of the Chat-BAC mice have the Chat gene disrupted by the fluorescent protein- or Cre-encoding gene but must be considered as transgenic for Slc18a3/VAChT (Tallini et al., 2006; von Engelhardt et al., 2007; Grybko et al., 2011; Zhao et al., 2011), unless this gene has been purposefully inactivated (Ting and Feng, 2014). The overexpression of VAChT in some of these mouse lines is reportedly associated with novel phenotypes (Nagy and Aubert, 2012, 2013, 2015; Kolisnyk et al., 2013; Crittenden et al., 2014). The Chat-BAC mouse lines show excellent and faithful transgene reporter expression in cholinergic cells, i.e., fluorescent proteins mark the appropriate neuronal populations (e.g., motor neurons, interneurons in the striatum, projection neurons of the medial habenula, and BFCN) and have the appropriate phenotypes including the expression of endogenous Chat as visualized by immunostaining. Thus, it is clear that studies of these neuronal populations provide information about the function and properties of bona fide cholinergic neurons. In our hands, one of these lines (B6.Cg-Tg(RP23-268L19EGFP)2Mik/J; Tallini et al., 2006) has permitted studies on BFCN purified by fluorescence-activated cell sorting (Schnitzler et al., 2008, 2010) and on the effects of growth factors on BFCN in culture (Schnitzler et al., 2010) as well as in vivo in transgenic mouse models of AD (Burke et al., 2013; Mellott et al., 2014). Unfortunately, transgene reporter expression in Chat-BAC mouse lines is sometimes seen in brain regions not known for the presence of cholinergic neurons, such as the hippocampus. Figure 1 shows an example from this laboratory. We used the B6.Cg-Tg(RP23-268L19-EGFP)2Mik/J mice and examined ChAT-immunofluorescence and native EGFP fluorescence by confocal microscopy in brain sections. We found excellent colocalization of the EGFP and ChAT signals in the neurons and neuronal processes in septal BFCN. In contrast, the fluorescent EGFP-expressing cells in the hippocampus showed minimal, or no ChAT staining, and their processes were devoid of the ChAT signal even though most of the processes in this region showed colocalization of ChAT and EGFP, indicating that our immunostaining has adequate sensitivity 

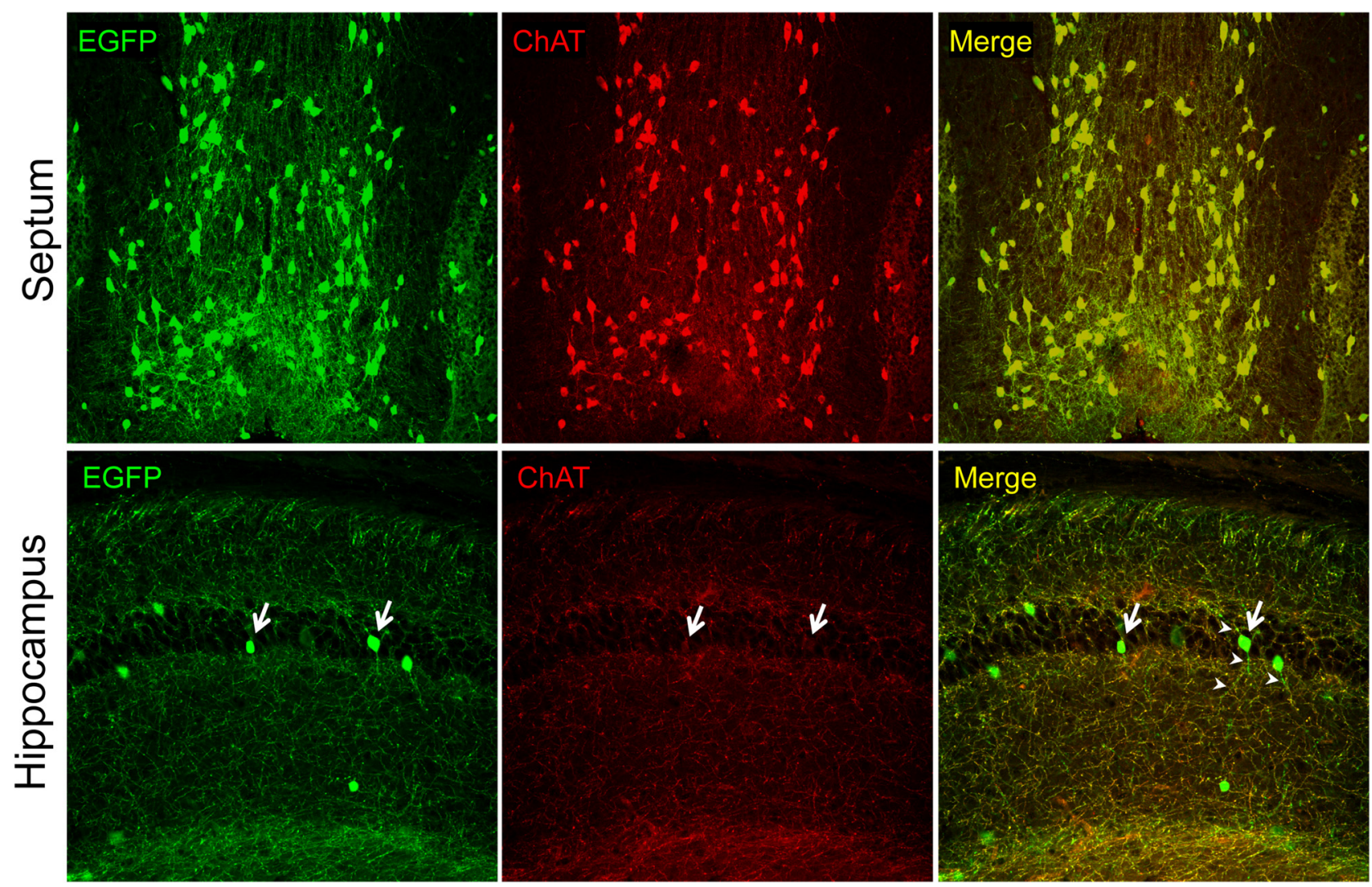

FIGURE 1 | Intrinsic EGFP fluorescence and choline acetyltransferase (ChAT) immunofluorescence (Millipore AB144P; 1:200) in brain sections of the 21 days old ChAT-EGFP mice visualized with confocal microscopy. Note, strong ChAT staining and colocalization of the EGFP and ChAT signal (red) in the septal cell bodies and fibers (top panels). In contrast, the hippocampus (bottom panels) shows minimal (arrows) or no ChAT cell body staining and the colocalization of the EGFP and ChAT signals is seen only in fibers that do not emanate from the hippocampal cell bodies (presumably the septohippocampal afferents). The fibers of the EGFP-expressing cells do not show ChAT-immunostaining (arrow heads). Original magnifications: 10x (top panels) and 20× (lower panels). All procedures were performed in accordance with the protocol approved by the Boston University IACUC.

for the detection of ChAT even in small structures. The latter fibers undoubtedly represent the septohippocampal afferents. In a report published recently, Yi et al. (2015) examined the electrophysiological and molecular phenotypes of fluorescent hippocampal cells using Chat-EGFP and Chat-Cre (crossed with Rosa26EYFP mice to generate EYFP-expressing cells) mouse lines; see also the associated editorial by Vijayaraghavan and Sharma (2015). They appropriately used quotation marks when referring to these cells as "cholinergic neurons" because while the cells exhibited the reporter protein expression, they did not stain for ChAT, indicating that they did not produce ACh. Moreover, the authors were unable to show ACh release from these cells. Instead these "cholinergic neurons" released glutamate upon depolarization and thus must be considered as glutamatergic. We conclude that the data reported by $\mathrm{Yi}$ et al. (2015), together with our observations (Figure 1), indicate that the adult hippocampus is devoid of intrinsic cholinergic neurons, consistent with the studies cited above. Whether such neurons occur early in development remains to be determined. If they do however, one would expect a relatively high number of EYFP-expressing hippocampal cells to arise from the Cremediated recombination in the Chat-Cre $\times$ Rosa26EYFP crosses performed by Yi et al. (2015). This, however was not observed, indicating that inappropriate activity of the Chat-BAC promoter constitutes a rare event in these mice, as acknowledged by Yi et al. (2015). The ectopic expression of the promoter may suggest that the BACs used for the generation of these mice are too short and do not contain all of the cis-DNA segments necessary for the appropriate silencing of the cholinergic gene locus in all non-cholinergic cells, or that the chromatin elements in the regions of BAC integration within the murine genome can occasionally override the normal control of the cholinergic gene locus. We note that the Chat-BAC mouse lines frequently harbor multiple copies of the $\mathrm{BAC}$ and that, to date, no studies on the integration sites of any of these BACs have been reported. Some of the drawbacks of the BAC-mediated transgenesis can be obviated by a knock-in strategy to produce comparable transgenic mice. Successful examples of this approach are lines in which a Cre allele, that is expressed via an internal ribosome entry site, was inserted 
downstream of the stop codon of the endogenous Chat gene as described by Rotolo et al. (2008) and Rossi et al. (2011). The former line has been used to investigate hippocampal cholinergic innervation. Consistent with the results of studies obtained with immunohistochemical methods, no intrinsic hippocampal cholinergic cells have been reported in these mice (Wu et al., 2014). In sum, the experience of the studies of Chat-BAC transgenic mice indicates that they are an excellent tool for the determination of the properties and physiological functions of bona fide cholinergic neurons in health and disease. However, their use to discover novel populations of cholinergic cells requires caution and rigor. If the only evidence for a new class of cholinergic neurons is the expression of a reporter protein in a Chat-BAC mouse line, it is best to

\section{REFERENCES}

Aimone, J. B., Li, Y., Lee, S. W., Clemenson, G. D., Deng, W., and Gage, F. H. (2014). Regulation and function of adult neurogenesis: from genes to cognition. Physiol. Rev. 94, 991-1026. doi: 10.1152/physrev.00004.2014

Armstrong, D. M., Saper, C. B., Levey, A. I., Wainer, B. H., and Terry, R. D. (1983). Distribution of cholinergic neurons in rat brain: demonstrated by the immunocytochemical localization of choline acetyltransferase. J. Comp. Neurol. 216, 53-68. doi: 10.1002/cne.902160106

Augustinack, J. C., Van Der Kouwe, A. J., Salat, D. H., Benner, T., Stevens, A. A., Annese, J., et al. (2014). H.M.'s contributions to neuroscience: a review and autopsy studies. Hippocampus 24, 1267-1286. doi: 10.1002/hipo. 22354

Aztiria, E., Capodieci, G., Arancio, L., and Leanza, G. (2007). Extensive training in a maze task reduces neurogenesis in the adult rat dentate gyrus probably as a result of stress. Neurosci. Lett. 416, 133-137. doi: 10.1016/j.neulet.2007. 01.069

Berse, B., and Blusztajn, J. K. (1995). Co-ordinated up-regulation of choline acetyltransferase and vesicular acetylcholine transporter gene expression by the retinoic acid receptor alpha, CAMP and CNTF/LIF signaling pathways in a murine septal cell line. J. Biol. Chem. 270, 22101-22104. doi: 10.1074/jbc.270. 38.22101

Bird, C. M., and Burgess, N. (2008). The hippocampus and memory: insights from spatial processing. Nat. Rev. Neurosci. 9, 182-194. doi: 10.1038/nrn2335

Blaker, S. N., Armstrong, D. M., and Gage, F. H. (1988). Cholinergic neurons within the rat hippocampus: response to fimbria-fornix transection. J. Comp. Neurol. 272, 127-138. doi: 10.1002/cne.902720109

Bowen, D. M., Allen, S. J., Benton, J. S., Goodhardt, M. J., Haan, E. A., Palmer, A. M., et al. (1983). Biochemical assessment of serotonergic and cholinergic dysfunction and cerebral atrophy in Alzheimer's disease. J. Neurochem. 41, 266-272. doi: 10.1111/j.1471-4159.1983.tb11838.x

Burke, R. M., Norman, T. A., Haydar, T. F., Slack, B. E., Leeman, S. E., Blusztajn, J. K., et al. (2013). BMP9 ameliorates amyloidosis and the cholinergic defect in a mouse model of Alzheimer's disease. Proc. Natl. Acad. Sci. U S A 110, 19567-19572. doi: 10.1073/pnas.1319297110

Clarke, D. J. (1985). Cholinergic innervation of the rat dentate gyrus: an immunocytochemical and electron microscopical study. Brain Res. 360, 349-354. doi: 10.1016/0006-8993(85)91253-3

Cooper-Kuhn, C. M., Winkler, J., and Kuhn, H. G. (2004). Decreased neurogenesis after cholinergic forebrain lesion in the adult rat. J. Neurosci. Res. 77, 155-165. doi: 10.1002/jnr.20116

Crittenden, J. R., Lacey, C. J., Lee, T., Bowden, H. A., and Graybiel, A. M. (2014). Severe drug-induced repetitive behaviors and striatal overexpression of VAChT in ChAT-ChR2-EYFP BAC transgenic mice. Front. Neural Circuits 8:57. doi: 10.3389/fncir.2014.00057

Eiden, L. E. (1998). The cholinergic gene locus. J. Neurochem. 70, 2227-2240. doi: 10.1046/j.1471-4159.1998.70062227.x

Fontana, X., Nácher, J., Soriano, E., and del Réo, J. A. (2006). Cell proliferation in the adult hippocampal formation of rodents and its modulation by conclude that such a class does not exist. Because there are now many BAC transgenic lines available to investigators, we posit that similar considerations should be applied to those.

\section{AUTHOR CONTRIBUTIONS}

JKB conceived and wrote the manuscript. JR performed the study and wrote the manuscript.

\section{FUNDING}

Supported by an NIH grant AG045031. JR was supported by the Austrian Marshall Plan Foundation.

entorhinal and fimbria-fornix afferents. Cereb. Cortex 16, 301-312. doi: 10 . 1093/cercor/bhi120

Fréchette, M., Rennie, K., and Pappas, B. A. (2009). Developmental forebrain cholinergic lesion and environmental enrichment: behaviour, CA1 cytoarchitecture and neurogenesis. Brain Res. 1252, 172-182. doi: 10. 1016/j.brainres.2008.11.082

Frotscher, M., Schlander, M., and Léránth, C. (1986). Cholinergic neurons in the hippocampus. A combined light- and electron-microscopic immunocytochemical study in the rat. Cell Tissue Res. 246, 293-301. doi: 10. 1007/bf00215891

Gauthier, S., Reisberg, B., Zaudig, M., Petersen, R. C., Ritchie, K., Broich, K., et al. (2006). Mild cognitive impairment. Lancet 367, 1262-1270. doi: 10 1016/S0140-6736(06)68542-5

Gilmor, M. L., Counts, S. E., Wiley, R. G., and Levey, A. I. (1998). Coordinate expression of the vesicular acetylcholine transporter and choline acetyltransferase following septohippocampal pathway lesion. J. Neurochem. 71, 2411-2420. doi: 10.1046/j.1471-4159.1998. 71062411.x

Gilmor, M. L., Nash, N. R., Roghani, A., Edwards, R. H., Yi, H., Hersch, S. M., et al. (1996). Expression of the putative vesicular acetylcholine transporter in rat brain and localization in cholinergic synaptic vesicles. J. Neurosci. 16, 2179-2190.

Gong, S., Doughty, M., Harbaugh, C. R., Cummins, A., Hatten, M. E., Heintz, N., et al. (2007). Targeting Cre recombinase to specific neuron populations with bacterial artificial chromosome constructs. J. Neurosci. 27, 9817-9823. doi: 10. 1523/JNEUROSCI.2707-07.2007

Gong, S., Zheng, C., Doughty, M. L., Losos, K., Didkovsky, N., Schambra, U. B., et al. (2003). A gene expression atlas of the central nervous system based on bacterial artificial chromosomes. Nature 425, 917-925. doi: 10. 1038/nature02033

Goto, Y., Niidome, T., Hongo, H., Akaike, A., Kihara, T., and Sugimoto, H. (2008). Impaired muscarinic regulation of excitatory synaptic transmission in the APPswe/PS1dE9 mouse model of Alzheimer's disease. Eur. J. Pharmacol. 583, 84-91. doi: 10.1016/j.ejphar.2008.01.030

Gray, D. T., and Barnes, C. A. (2015). Distinguishing adaptive plasticity from vulnerability in the aging hippocampus. Neuroscience 309, 17-28. doi: 10 . 1016/j.neuroscience.2015.08.001

Grothe, M., Heinsen, H., and Teipel, S. J. (2012). Atrophy of the cholinergic Basal forebrain over the adult age range and in early stages of Alzheimer's disease. Biol. Psychiatry 71, 805-813. doi: 10.1016/j.biopsych.2011. 06.019

Grybko, M. J., Hahm, E. T., Perrine, W., Parnes, J. A., Chick, W. S., Sharma, G., et al. (2011). A transgenic mouse model reveals fast nicotinic transmission in hippocampal pyramidal neurons. Eur. J. Neurosci. 33, 1786-1798. doi: 10. 1111/j.1460-9568.2011.07671.x

Haense, C., Kalbe, E., Herholz, K., Hohmann, C., Neumaier, B., Krais, R., et al. (2012). Cholinergic system function and cognition in mild cognitive impairment. Neurobiol. Aging 33, 867-877. doi: 10.1016/j.neurobiolaging.2010. 08.015 
Heintz, N. (2004). Gene expression nervous system atlas (GENSAT). Nat. Neurosci. 7:483. doi: 10.1038/nn0504-483

Ho, N. F., Han, S. P., and Dawe, G. S. (2009). Effect of voluntary running on adult hippocampal neurogenesis in cholinergic lesioned mice. BMC Neurosci. 10:57. doi: 10.1186/1471-2202-10-57

Ichikawa, T., Ajiki, K., Matsuura, J., and Misawa, H. (1997). Localization of two cholinergic markers, choline acetyltransferase and vesicular acetylcholine transporter in the central nervous system of the rat: in situ hybridization histochemistry and immunohistochemistry. J. Chem. Neuroanat. 13, 23-39. doi: 10.1016/s0891-0618(97)00021-5

Ichikawa, T., and Hirata, Y. (1986). Organization of choline acetyltransferasecontaining structures in the forebrain of the rat. J. Neurosci. 6, 281-292.

Itou, Y., Nochi, R., Kuribayashi, H., Saito, Y., and Hisatsune, T. (2011). Cholinergic activation of hippocampal neural stem cells in aged dentate gyrus. Hippocampus 21, 446-459. doi: 10.1002/hipo.20761

Kanaya-Ida, S., and Ben Ari, Y. (1989). Transient increase in the number of cholinergic neurons in the developing rat dentate gyrus. Neurosci. Lett. 101, 23-28. doi: 10.1016/0304-3940(89)90434-5

Kaneko, N., Okano, H., and Sawamoto, K. (2006). Role of the cholinergic system in regulating survival of newborn neurons in the adult mouse dentate gyrus and olfactory bulb. Genes Cells 11, 1145-1159. doi: 10.1111/j.1365-2443.2006. 01010.x

Kolisnyk, B., Guzman, M. S., Raulic, S., Fan, J., Magalhães, A. C., Feng, G., et al. (2013). ChAT-ChR2-EYFP mice have enhanced motor endurance but show deficits in attention and several additional cognitive domains. J. Neurosci. 33, 10427-10438. doi: 10.1523/JNEUROSCI.0395-13.2013

Kotani, S., Yamauchi, T., Teramoto, T., and Ogura, H. (2006). Pharmacological evidence of cholinergic involvement in adult hippocampal neurogenesis in rats. Neuroscience 142, 505-514. doi: 10.1016/j.neuroscience.2006.06.035

Kotani, S., Yamauchi, T., Teramoto, T., and Ogura, H. (2008). Donepezil, an acetylcholinesterase inhibitor, enhances adult hippocampal neurogenesis. Chem. Biol. Interact. 175, 227-230. doi: 10.1016/j.cbi.2008.04.004

Lauterborn, J. C., Isackson, P. J., Montalvo, R., and Gall, C. M. (1993). In situ hybridization localization of choline acetyltransferase mRNA in adult rat brain and spinal cord. Mol. Brain Res. 17, 59-69. doi: 10.1016/0169-328x(93) 90073-X

Lee, S. W., Clemenson, G. D., and Gage, F. H. (2012). New neurons in an aged brain. Behav. Brain Res. 227, 497-507. doi: 10.1016/j.bbr.2011. 10.009

Machová, E., Rudajev, V., Smycková, H., Koivisto, H., Tanila, H., and Dolezal, V. (2010). Functional cholinergic damage develops with amyloid accumulation in young adult APPswe/PS1dE9 transgenic mice. Neurobiol. Dis. 38, 27-35. doi: 10.1016/j.nbd.2009.12.023

Matthews, D. A., Salvaterra, P. M., Crawford, G. D., Houser, C. R., and Vaughn, J. E. (1987). An immunocytochemical study of choline acetyltransferase-containing neurons and axon terminals in normal and partially deafferented hippocampal formation. Brain Res. 402, 30-43. doi: 10. 1016/0006-8993(87)91044-4

Mellott, T. J., Pender, S. M., Burke, R. M., Langley, E. A., and Blusztajn, J. K. (2014). IGF2 ameliorates amyloidosis, increases cholinergic marker expression and raises BMP9 and neurotrophin levels in the hippocampus of the APPswePS1dE9 Alzheimer's disease model mice. PLoS One 9:e94287. doi: 10. 1371/journal.pone.0094287

Mohapel, P., Leanza, G., Kokaia, M., and Lindvall, O. (2005). Forebrain acetylcholine regulates adult hippocampal neurogenesis and learning. Neurobiol. Aging 26, 939-946. doi: 10.1016/j.neurobiolaging.2004. 07.015

Mufson, E. J., Counts, S. E., Perez, S. E., and Ginsberg, S. D. (2008). Cholinergic system during the progression of Alzheimer's disease: therapeutic implications. Expert Rev. Neurother. 8, 1703-1718. doi: 10.1586/14737175.8.11.1703

Nagy, P. M., and Aubert, I. (2012). Overexpression of the vesicular acetylcholine transporter increased acetylcholine release in the hippocampus. Neuroscience 218, 1-11. doi: 10.1016/j.neuroscience.2012.05.047

Nagy, P. M., and Aubert, I. (2013). B6eGFPChAT mice overexpressing the vesicular acetylcholine transporter exhibit spontaneous hypoactivity and enhanced exploration in novel environments. Brain Behav. 3, 367-383. doi: 10. $1002 /$ brb3.139
Nagy, P. M., and Aubert, I. (2015). Overexpression of the vesicular acetylcholine transporter enhances dendritic complexity of adult-born hippocampal neurons and improves acquisition of spatial memory during aging. Neurobiol. Aging 36, 1881-1889. doi: 10.1016/j.neurobiolaging.2015.02.021

Narimatsu, N., Harada, N., Kurihara, H., Nakagata, N., Sobue, K., and Okajima, K. (2009). Donepezil improves cognitive function in mice by increasing the production of insulin-like growth factor-I in the hippocampus. J. Pharmacol. Exp. Ther. 330, 2-12. doi: 10.1124/jpet.108.147280

Nikolajsen, G. N., Jensen, M. S., and West, M. J. (2011). Cholinergic axon length reduced by 300 meters in the brain of an Alzheimer mouse model. Neurobiol. Aging 32, 1927-1931. doi: 10.1016/j.neurobiolaging.2011.05.006

Oh, J. D., Woolf, N. J., Roghani, A., Edwards, R. H., and Butcher, L. L. (1992). Cholinergic neurons in the rat central nervous system demonstrated by in situ hybridization of choline acetyltransferase mRNA. Neuroscience 47, 807-822. doi: 10.1016/0306-4522(92)90031-v

Payette, D. J., Xie, J., and Guo, Q. (2007). Reduction in CHT1-mediated choline uptake in primary neurons from presenilin-1 M146V mutant knock-in mice. Brain Res. 1135, 12-21. doi: 10.1016/j.brainres.2006.12.005

Perez, S. E., Dar, S., Ikonomovic, M. D., DeKosky, S. T., and Mufson, E. J. (2007). Cholinergic forebrain degeneration in the APPswe/PS1DeltaE9 transgenic mouse. Neurobiol. Dis. 28, 3-15. doi: 10.1016/j.nbd.2007.06.015

Rennie, K., Fréchette, M., and Pappas, B. A. (2011). The effects of neonatal forebrain cholinergic lesion on adult hippocampal neurogenesis. Brain Res. 1373, 79-90. doi: 10.1016/j.brainres.2010.11.091

Roghani, A., Shirzadi, A., Butcher, L. L., and Edwards, R. H. (1998). Distribution of the vesicular transporter for acetylcholine in the rat central nervous system. Neuroscience 82, 1195-1212. doi: 10.1016/s0306-4522(97)00291-1

Roghani, A., Shirzadi, A., Kohan, S. A., Edwards, R. H., and Butcher, L. L. (1996). Differential distribution of the putative vesicular transporter for acetylcholine in the rat central nervous system. Mol. Brain Res. 43, 65-76. doi: 10.1016/s0169$328 \mathrm{x}(96) 00145-3$

Rossi, J., Balthasar, N., Olson, D., Scott, M., Berglund, E., Lee, C. E., et al. (2011). Melanocortin-4 receptors expressed by cholinergic neurons regulate energy balance and glucose homeostasis. Cell Metab. 13, 195-204. doi: 10.1016/j.cmet. 2011.01.010

Rotolo, T., Smallwood, P. M., Williams, J., and Nathans, J. (2008). Geneticallydirected, cell type-specific sparse labeling for the analysis of neuronal morphology. PLoS One 3:e4099. doi: 10.1371/journal.pone.0004099

Samson, R. D., and Barnes, C. A. (2013). Impact of aging brain circuits on cognition. Eur. J. Neurosci. 37, 1903-1915. doi: 10.1111/ejn.12183

Sarter, M., and Bruno, J. P. (2004). Developmental origins of the agerelated decline in cortical cholinergic function and associated cognitive abilities. Neurobiol. Aging 25, 1127-1139. doi: 10.1016/j.neurobiolaging.2003. 11.011

Savonenko, A., Xu, G. M., Melnikova, T., Morton, J. L., Gonzales, V., Wong, M. P., et al. (2005). Episodic-like memory deficits in the APPswe/PS1dE9 mouse model of Alzheimer's disease: relationships to beta-amyloid deposition and neurotransmitter abnormalities. Neurobiol. Dis. 18, 602-617. doi: 10.1016/j. nbd.2004.10.022

Schäfer, M. K. H., Eiden, L. E., and Weihe, E. (1998). Cholinergic neurons and terminal fields revealed by immunohistochemistry for the vesicular acetylcholine transporter. I. Central nervous system. Neuroscience 84, 331-359. doi: 10.1016/s0306-4522(97)00516-2

Schnitzler, A. C., Lopez-Coviella, I., and Blusztajn, J. K. (2008). Purification and culture of nerve growth factor receptor (p75)-expressing basal forebrain cholinergic neurons. Nat. Protoc. 3, 34-40. doi: 10.1038/nprot.2007.477

Schnitzler, A. C., Mellott, T. J., Lopez-Coviella, I., Tallini, Y. N., Kotlikoff, M. I., Follettie, M. T., et al. (2010). BMP9 (bone morphogenetic protein 9) induces NGF as an autocrine/paracrine cholinergic trophic factor in developing basal forebrain neurons. J. Neurosci. 30, 8221-8228. doi: 10.1523/jneurosci.5611-09. 2010

Tallini, Y. N., Shui, B., Greene, K. S., Deng, K. Y., Doran, R., Fisher, P. J., et al. (2006). BAC transgenic mice express enhanced green fluorescent protein in central and peripheral cholinergic neurons. Physiol. Genomics 27, 391-397. doi: 10.1152/physiolgenomics.00092.2006

Teles-Grilo Ruivo, L. M., and Mellor, J. R. (2013). Cholinergic modulation of hippocampal network function. Front. Synaptic Neurosci. 5:2. doi: 10. 3389/fnsyn.2013.00002 
Ting, J. T., and Feng, G. (2014). Recombineering strategies for developing next generation BAC transgenic tools for optogenetics and beyond. Front. Behav. Neurosci. 8:111. doi: 10.3389/fnbeh.2014.00111

Van der Borght, K., Mulder, J., Keijser, J. N., Eggen, B. J., Luiten, P. G., and Van der Zee, E. A. (2005). Input from the medial septum regulates adult hippocampal neurogenesis. Brain Res. Bull. 67, 117-125. doi: 10.1016/j.brainresbull.2005. 06.018

Van Kampen, J. M., and Eckman, C. B. (2010). Agonist-induced restoration of hippocampal neurogenesis and cognitive improvement in a model of cholinergic denervation. Neuropharmacology 58, 921-929. doi: 10.1016/j. neuropharm.2009.12.005

Vijayaraghavan, S., and Sharma, G. (2015). Editorial: brain cholinergic mechanisms. Front. Synaptic Neurosci. 7:14. doi: 10.3389/fnsyn.2015.00014

von Engelhardt, J., Eliava, M., Meyer, A. H., Rozov, A., and Monyer, H. (2007). Functional characterization of intrinsic cholinergic interneurons in the cortex. J. Neurosci. 27, 5633-5642. doi: 10.1523/jneurosci.4647-06.2007

Wainer, B. H., Levey, A. I., Rye, D. B., Mesulam, M. M., and Mufson, E. J. (1985). Cholinergic and non-cholinergic septohippocampal pathways. Neurosci. Lett. 54, 45-52. doi: 10.1016/s0304-3940(85)80116-6

Whitehouse, P. J., Price, D. L., Struble, R. G., Clark, A. W., Coyle, J. T., and Delong, M. R. (1982). Alzheimer's disease and senile dementia: loss of neurons in the basal forebrain. Science 215, 1237-1239. doi: 10.1126/science.7058341

Wu, H., Williams, J., and Nathans, J. (2014). Complete morphologies of basal forebrain cholinergic neurons in the mouse. Elife 3:e02444. doi: 10.7554/elife. 02444
Yi, F., Catudio-Garrett, E., Gábriel, R., Wilhelm, M., Erdelyi, F., Szabo, G., et al. (2015). Hippocampal "cholinergic interneurons" visualized with the choline acetyltransferase promoter: anatomical distribution, intrinsic

membrane properties, neurochemical characteristics and capacity for cholinergic modulation. Front. Synaptic Neurosci. 7:4. doi: 10.3389/fnsyn.2015. 00004

Zhao, S., Ting, J. T., Atallah, H. E., Qiu, L., Tan, J., Gloss, B., et al. (2011). Cell type-specific channelrhodopsin-2 transgenic mice for optogenetic dissection of neural circuitry function. Nat. Methods 8, 745-752. doi: 10.1038/nmeth.1668

Zhao, N., Zhong, C., Wang, Y., Zhao, Y., Gong, N., Zhou, G., et al. (2008). Impaired hippocampal neurogenesis is involved in cognitive dysfunction induced by thiamine deficiency at early pre-pathological lesion stage. Neurobiol. Dis. 29, 176-185. doi: 10.1016/j.nbd.2007.08.014

Conflict of Interest Statement: The authors declare that the research was conducted in the absence of any commercial or financial relationships that could be construed as a potential conflict of interest.

Copyright (C) 2016 Blusztajn and Rinnofner. This is an open-access article distributed under the terms of the Creative Commons Attribution License (CC BY). The use, distribution and reproduction in other forums is permitted, provided the original author(s) or licensor are credited and that the original publication in this journal is cited, in accordance with accepted academic practice. No use, distribution or reproduction is permitted which does not comply with these terms. 